\title{
A compact micro-bolometer array for mid-infrared laser beam alignment, diagnostics and spot-size measurement
}

\author{
IP Clark and M Towrie \\ Science and Technology Facilities Council, Central Laser Facility, Research Complex at Harwell, Harwell \\ Campus, Didcot, Oxfordshire, OX11 OQX, UK \\ a) Author to whom correspondence should be addressed: ian.p.clark@stfc.ac.uk
}

Knowledge of a lasers beam's profile throughout a laser system and experiment can help immensely in diagnosing laser problems and assisting in beam alignment and focusing at a sample. Obtaining such profiles is a trivial task in the ultraviolet-visible wavelength range but more challenging with near-infrared to infrared beams. Scientific grade bolometer arrays, suitable for such a task, do exist but are extremely costly, relatively large and have a large pixel size, of the order of $80 \mu \mathrm{m}$, which is adequate for profiling larger beams but poses an issue when trying to profile sub $100 \mu \mathrm{m}$ beams for example at a focal point.

This communication identifies a micro-bolometer array for near- to mid-infrared laser beam profiling, which is extremely low cost. In addition, the device is very compact, enabling use in confined spaces, and has a small, 12 $\mu \mathrm{m}$, pixel size permitting the profiling of focused laser beams. The best scientific grade device identified has a pixel size of $17 \mu \mathrm{m}$. This device is a powerful tool for infrared laser spectroscopists, reducing the time required to measure the spot size of beams and to achieve spatial overlap of multiple infrared beams as used in twodimensional infrared spectroscopy, saving many hours of setup time. The use of the bolometer array as a spectrographic detector and probe of long-term beam drifts is also demonstrated.

\section{Introduction}

The intensity of light impinging on a sample and the spot-size of beams at the sample are two of the most important factors influencing the collection of quantitative data in many scientific disciplines, but particularly in laserbased time-resolved spectroscopic applications. The operator needs to know the spot-size and power of both pump and probe beams incident on the sample. It is a trivial task, not discussed here, to measure the power of laser beams using a power meter. Recording the spot-size of laser beams, in particular in the mid-IR, at the target is however, less trivial. A number of methods are available to the researcher including visual inspection of laser burn spots, knife-edge, pinhole, or slit-method and array camera methods. These techniques are reviewed in references [1] and [2]. 
Visual methods of analysing a beam profile, whilst simple and low cost, are somewhat crude and subjective. The human eye perceives brightness as a logarithmic function of the light intensity entering the eye and is limited to perceiving approximately 30 shades of grey.[3] A study by Kimpe and Tuytschaever [4] shows that given the right conditions, e.g. a high specification medical display, the human visual system is potentially capable of observing approximately 900 shades of grey. Assuming the eye can perceive such a high number of grey shades, viewing a laser burn pattern has the disadvantage of only being practical for larger spot sizes, not sub-millimetre beam sizes, and only being useful with beams of high enough energy to induce a burn. When attempting to determine the size of a focal spot, the other non-digital methods such as the use of a knife-edge, slit or pinhole, are time consuming requiring the operator to traverse the device through the beam and along the optical axis to locate the focal point.

The location in space of infrared beams is also a critical factor to collecting quality data in infrared spectroscopy, with some techniques such as two-dimensional infrared spectroscopy requiring not only temporal overlap of two or more beams but also spatial overlap. At present commercial scientific infrared cameras, which can aid in both spotsize and beam overlap are beyond the reach of many budgets, and often have pixel sizes approaching or greater than the focused infrared beams used in such experiments. Thus not only are the cameras expensive they are far from optimal for small diameter beams. Another issue that may hinder their use, depending on the experimental arrangement, is the large size of these devices, which can be up to $80 \times 80 \times 40 \mathrm{~mm}$, a volume which is further enlarged by the space required for power and communication cables.

We present an ultra-compact and, commercially available micro-bolometer array thermal camera with a small pixel size, which can be adapted for the purpose of profiling beams (up to approximately $0.5 \mathrm{~mm}$ in diameter) in the near to mid-infrared range. The device has the potential to reduce significantly the setup time of any spectroscopic technique requiring the spot-size measurement of infrared beams or requiring spatial overlap of multiple infrared beams and has many other laboratory uses.

\section{Experimental}

A micro-bolometer array meeting the following criteria: commercially available, low cost, compact and with small pixel size was identified and purchased. The device (Seek Thermal, model Compact XR Android) is $45 \times 25 \times 18 \mathrm{~mm}$ in size and has a $206 \times 156$ element sensor array with a pixel pitch of $12 \mu \mathrm{m}$, manufactured by Raytheon. The array 
dimensions are approximately $2.5 \times 1.8 \mathrm{~mm}$. The micro-bolometer is equipped with an internal shutter, which periodically blocks the sensor during the cameras calibration process and has a micro-B USB 2.0 male connector for communication, being designed for connecting to Android phones. As purchased the device has an attached adjustable focus lens, which appears to be a made from a chalcogenide material and provides a $20^{\circ}$ field of view. The use of the lens clearly aids the collection of thermal images, which is the device's intended purpose, but prevents use in laser beam profiling applications. It was a relatively simple task, however, to remove the optic thus permitting a clear light path to the sensor.

Testing of the micro-bolometer was performed using the output of the Ultra LIFEtime system, which is part of the STFC's Central Laser Facility. References [5] and [6] give full details of this laser system and its capabilities. Briefly, the system consists of two synchronized $100 \mathrm{kHz}$ femtosecond lasers (Light Conversion, Pharos) and three optical parametric amplifiers (Light Conversion, Orpheus) providing one UV to mid-IR (210-16,000 nm) output and two independently tunable mid-IR (2,400-13,000 nm) outputs. The bolometer was located at the focal point of an off-axis parabolic mirror of focal length $75 \mathrm{~mm}$; all three of the laser systems infrared beams were incident on the mirror such that they were coincident at the focal point.

One of the infrared beams was diverted to a $125 \mathrm{~mm}$ spectrograph (Oriel Instruments, model 77400) with a 30 lines $/ \mathrm{mm}$ grating blazed at $8 \mu \mathrm{m}$ to test the use of the micro-bolometer as an infrared array detector for spectroscopic applications. A "chopped" mode of operation was used for these measurements, where by an image with and without the beam was recorded for each frame. Chopping was controlled by an in-house servo-motor based shutter controlled by an Arduino which linked to the data acquisition software.

In-house code written in the Python programming language enabled acquisition of the raw 14 bit greyscale data from the bolometer thus permitting easy processing, viewing and saving of images. All images reproduced are displayed using a perceptually uniform colour map, ie one in which equal data steps are perceived as equal steps in the colour space, and stored in ASCII format upon saving.

\section{Results and Discussions}

\section{Image Capture and Processing}

The Android version of the Seek bolometer purchased has a companion app available for download from the Google Play Store. The similar iPhone version (not tested) also has a companion app. Use of the app allows the 
lens-free device to operate as a beam profiler; however, it is obviously limited to a small format screen, lacks the software features often used during beam profiling such as $x$ - and $y$-cuts through the images and is a significant drain on the phone's battery.

The Seek bolometer array exports many frame modes, only two of which are understood - images with the shutter closed, to act as a background measurement, and images in 'normal' capture mode, the data from which can be processed as outlined below to yield usable images whether as an infrared camera for thermal imaging or a beam profiler as described here. The raw images from the array have every $15^{\text {th }}$ pixel set to zero; there is speculation on the web that this may be to avoid patent infringement.[7] Figure 1 shows a background image (a) and a raw 'normal mode' image (b) of the focal spot of a $6 \mu \mathrm{m}$ laser beam and a subtracted image, normal minus background, (c). The subtracted image still contains the artefacts at every $15^{\text {th }}$ pixel, which will hamper any beam profiling. These can however be corrected using a simple nonlinear digital filtering technique, in this case the application of a median filter with a window size of three, the result of this operation are shown in figure $1(\mathrm{~d})$. One downside of using a bolometer, which is essentially a thermal detector, is visible when comparing figure $1(\mathrm{a})$ and (b) - the background and raw images. With the device in a temperature-regulated laboratory at $20^{\circ} \mathrm{C}$ the background image intensity is approximately half the peak intensity of the pixels on which the beam is incident, which was recorded with the device close to saturation. This room temperature background essentially halves the usable dynamic range of the bolometer. The images are pixelated, a consequence of the beam spot size (full width at half maximum, FWHM) being ca 5 times the pixel size; a typical silicon based CCD sensor used for profiling UVvisible beams would have at least four times the pixel density. Other researchers have reported similar work for visible to near-infrared beams using web cameras as beam profilers which have a higher pixel density [8] and Langer and co-workers reported an elegant method for achieving sub-pixel resolution by operating in Bayermode.[9] This approach is not an option here as a micro-bolometer is not a colour device; however, the image can easily be enhanced, by resampling using a bicubic Mitchell Netravali image reconstruction filter with $B=C=1 / 3$, $[10,11]$ figure $1(d$, inset). Spot-size measurement, from $x$ - and $y$-cuts through the image, is carried out using the original, raw image. The data presented throughout the rest of this communication is raw data with no resampling. 

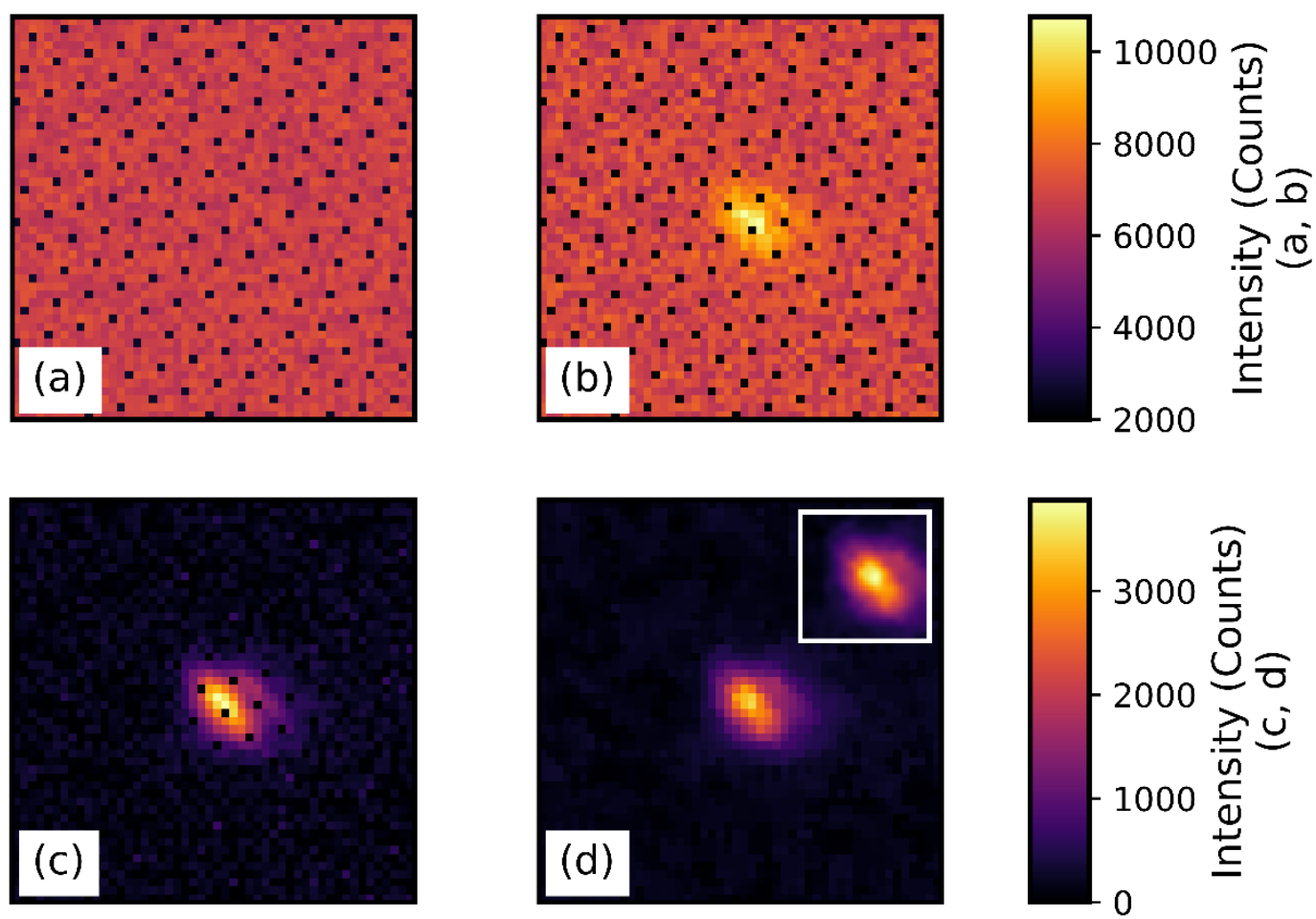

Figure 1. A single frame raw calibration (backgound) image (a) and 'normal' image (b) from the microbolometer array using a $6 \mu \mathrm{m}$ laser beam at $15 \mathrm{~nJ}$ and $5 \mathrm{kHz}$, due to the cameras frame rate the image is the result of ca 700 laser pulses. (c) shows the subtracted image, ie the calibration image subtracted from the 'normal' image. The image is enhanced by applying a median filter with a window size of three (d) and by resampling using a bicubic Mitchell-Netravali image reconstruction filter with $B=C=1 / 3$ (d, insert). Each image is of a $50 \times 50$ pixel section of the bolometer array.

Figure 2 shows cuts through the $x$ - and $y$-axes of the image displayed in figure $1(d)$. The captured data is of high enough quality to obtain laser spot-size measurements from Gaussian fits to the $x$-and y-cuts, figure 2(a) and (b). The cuts shown yield beam diameters, FWHM, of 90 and $77 \mu \mathrm{m}$ along the $\mathrm{x}$ - and $\mathrm{y}$-axes, with $\mathrm{R}^{2}$ values of 0.911 and 0.961 respectively, which is an indication of the quality of the laser beam spot rather than the bolometer array. Figure 2(c) illustrates the noise level of the device, showing the baseline across the whole of the $y$-cut, which excluding the peak has a mean of 152.5 and a standard deviation of 64.1 . 

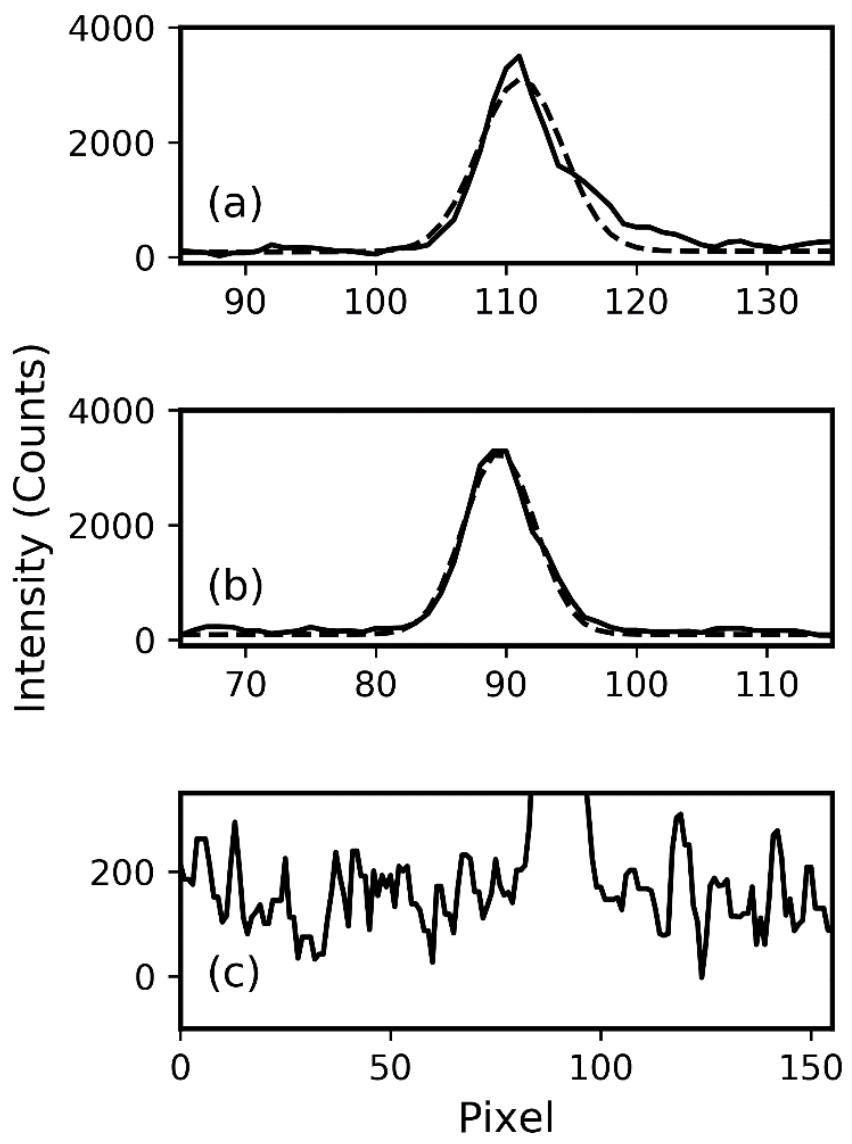

Figure 2. (a) $x$-cut through the image displayed in figure 1 (d) at y-pixel 90 and (b) $y$-cut through the same image at x-pixel 110. The broken lines in (a) and (b) are Gaussian fits to the x-and y-cuts respectively. (c) zoom into the baseline along the y-cut to illustrate the noise level of the micro-bolometer. Note, this data is from a single image frame which is the result of ca 700 laser pulses.

It is not possible to trigger the micro-bolometer from an external source so frame capture is limited to the devices inherent frame rate, which if simply acquiring and viewing images is ca $7 \mathrm{fps}$ using its USB 2.0 connection and a computer with Intel Core i5-3427U $1.8 \mathrm{GHz}$ processor and $6 \mathrm{~GB}$ RAM. If all images (raw, corrected and the corresponding calibration image) are saved for every frame this frame rate drops to ca $3.5 \mathrm{fps}$.

\section{Detector Response}

Possibly the most common detector used for infrared spectroscopic measurements is the mercury cadmium telluride, or MCT, detector, which typically peaks in spectral response around $10 \mu \mathrm{m}$, and has little response below $1 \mu \mathrm{m}$ or above $12 \mu \mathrm{m}$.[12] In general, a bolometer, which is a thermal detector, responds to a much broader wavelength range but is less sensitive, and typically optimized for the 8-14 $\mu \mathrm{m}$ thermal infrared atmospheric 
window.[13] An indication of the bolometer array's spectral response was obtained using a variety of wavelengths available from the Ultra laser system. Figure 3 shows the captured images, which were used to calculate a crude sensitivity at each wavelength (using the average power of the laser and the amplitude of a 2D-Gaussian peak fitted to the beam) using Equation 1, figure 4.

$$
\text { Sensitivity }=\frac{\text { Total intensity in FWHM }}{(\text { Number of pixels in FWHM } \times \text { Incident power })} \quad \text { Equation } 1
$$

As anticipated, the bolometer sensitivity is higher at $10 \mu \mathrm{m}$ than $1.3 \mu \mathrm{m}$, with sensitivity increasing through the mid-infrared region. What was not expected was the high sensitivity of the device at $1.6 \mu \mathrm{m}$. The exact cut on wavelength of the device, which is unable to see light in the visible region nor $800 \mathrm{~nm}$, is not known.

All the images above were recorded using beams with a pulse length of ca $180 \mathrm{fs}$. Although not shown here the bolometer was also tested, and found to function well, at $4 \mu \mathrm{m}$ with the nanosecond output of a periodically poled lithium niobate (Covesion) based optical parametric oscillator pumped by a 4 W 1064 nm diode-pumped solidstate laser (Wedge laser, Bright Solutions).
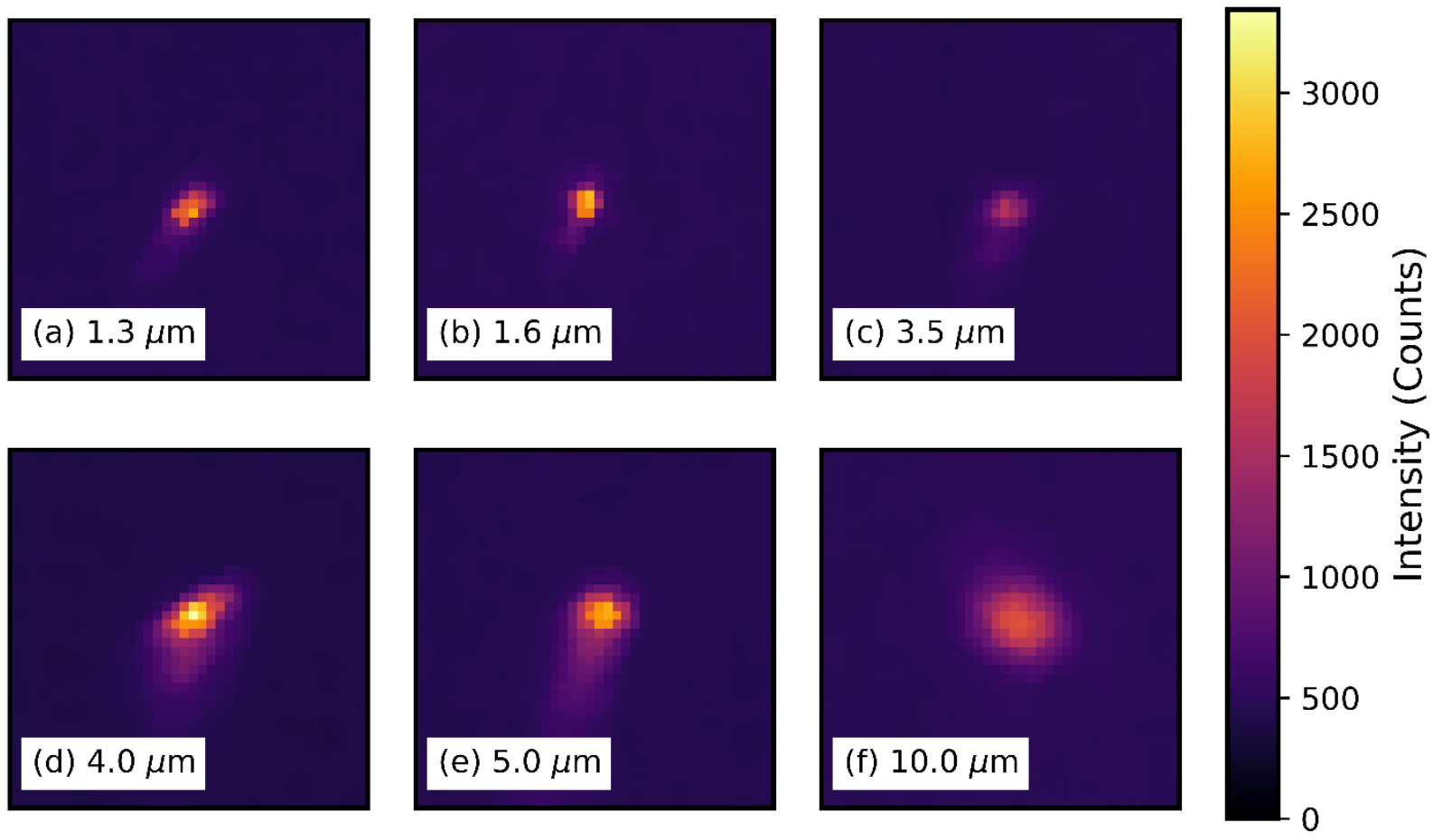

Figure 3. Images of near- and mid-infrared beams detected by the Seek Thermal micro-bolometer array. Each image is a $40 \times 40$ pixel segment of the total micro-bolometer array. (a) $1.3 \mu \mathrm{m}, 18 \mathrm{~nJ}$ at $6.25 \mathrm{kHz}$, ca 
890 pulses; (b) $1.6 \mu \mathrm{m}, 15 \mathrm{~nJ}$ at $0.2 \mathrm{kHz}$, ca 28 pulses; (c) $3.5 \mu \mathrm{m}, 10 \mathrm{~nJ}$ at $2 \mathrm{kHz}$, ca 290 pulses; (d) $4 \mu \mathrm{m}$, $20 \mathrm{~nJ}$ at $1.25 \mathrm{kHz}$, ca 180 pulses; (e) $5 \mu \mathrm{m}, 18 \mathrm{~nJ}$ at $1.25 \mathrm{kHz}$, ca 180 pulses; and (f) $10 \mu \mathrm{m}, 2.25 \mathrm{~nJ}$ at 0.1 kHz, ca 14 pulses.

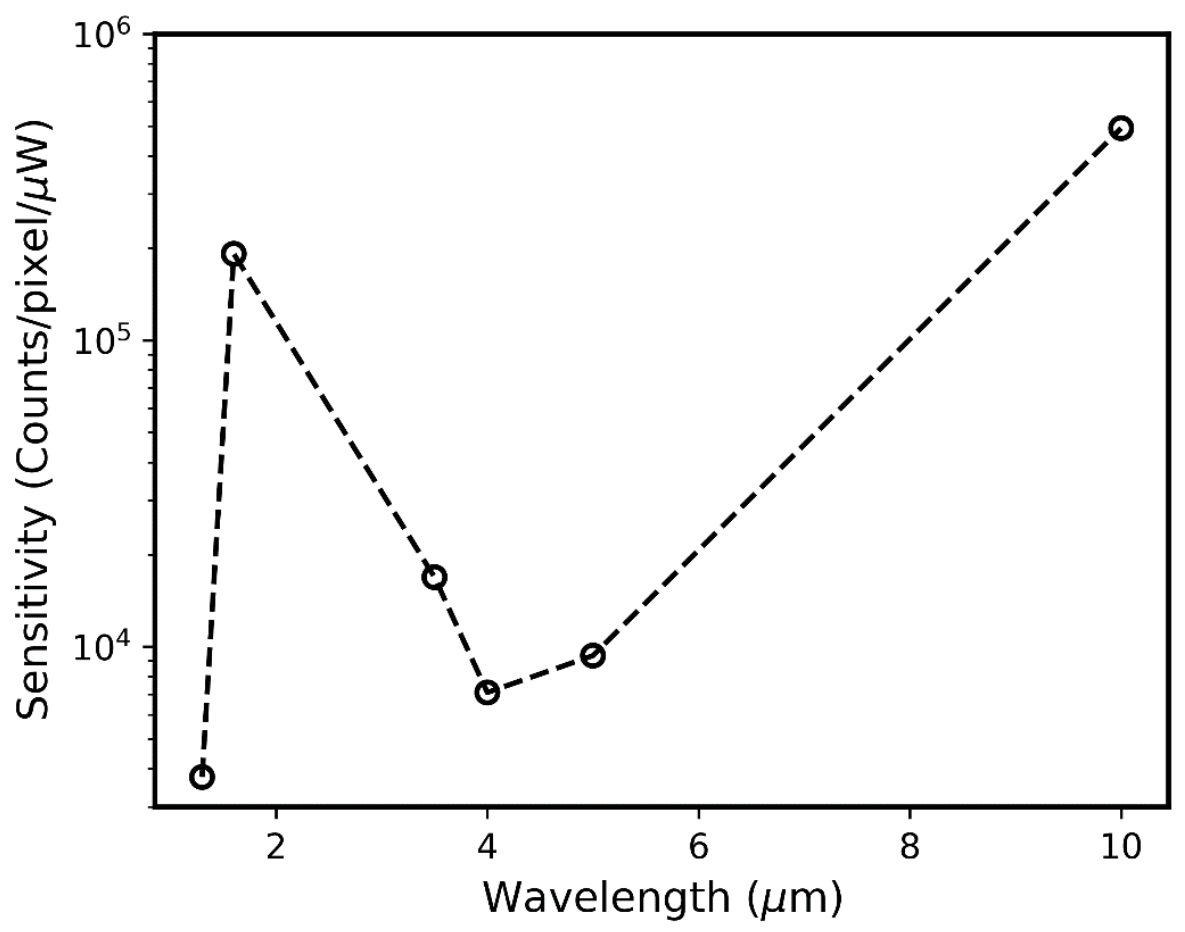

Figure 4. Plot of wavelength versus sensitivity of the bolometer array. Equation 1 was used to calculate sensitivity. Note, the ordinate is on a log scale.

The bolometer's linear dynamic range was measured using one of the infrared beams at $6 \mu \mathrm{m}$. The pulse picker on the laser provided an easy means of attenuating the average power by reducing its repetition rate; confirmation of the linearity of the power reduction with reduced repetition rate was obtained by measurement. Figure 5 shows a plot of the maximum intensity of the beam recorded in each image. The results show the 7,600 counts room temperature background and that in the intensity range 7,750 to 14,500 counts the response of the detector is linear, with a coefficient of determination, $R^{2}$, of 0.996 . Like any other photodetector, the spectral response is not flat, as observed in figure 3 and figure 4 . Whilst the observed intensity with a particular incident power changes with wavelength, we anticipate the intensity range over which the bolometer is linear to be the same. Although not tested, the micro-bolometers is likely to be sensitive up to approximately $20 \mu \mathrm{m}$. 


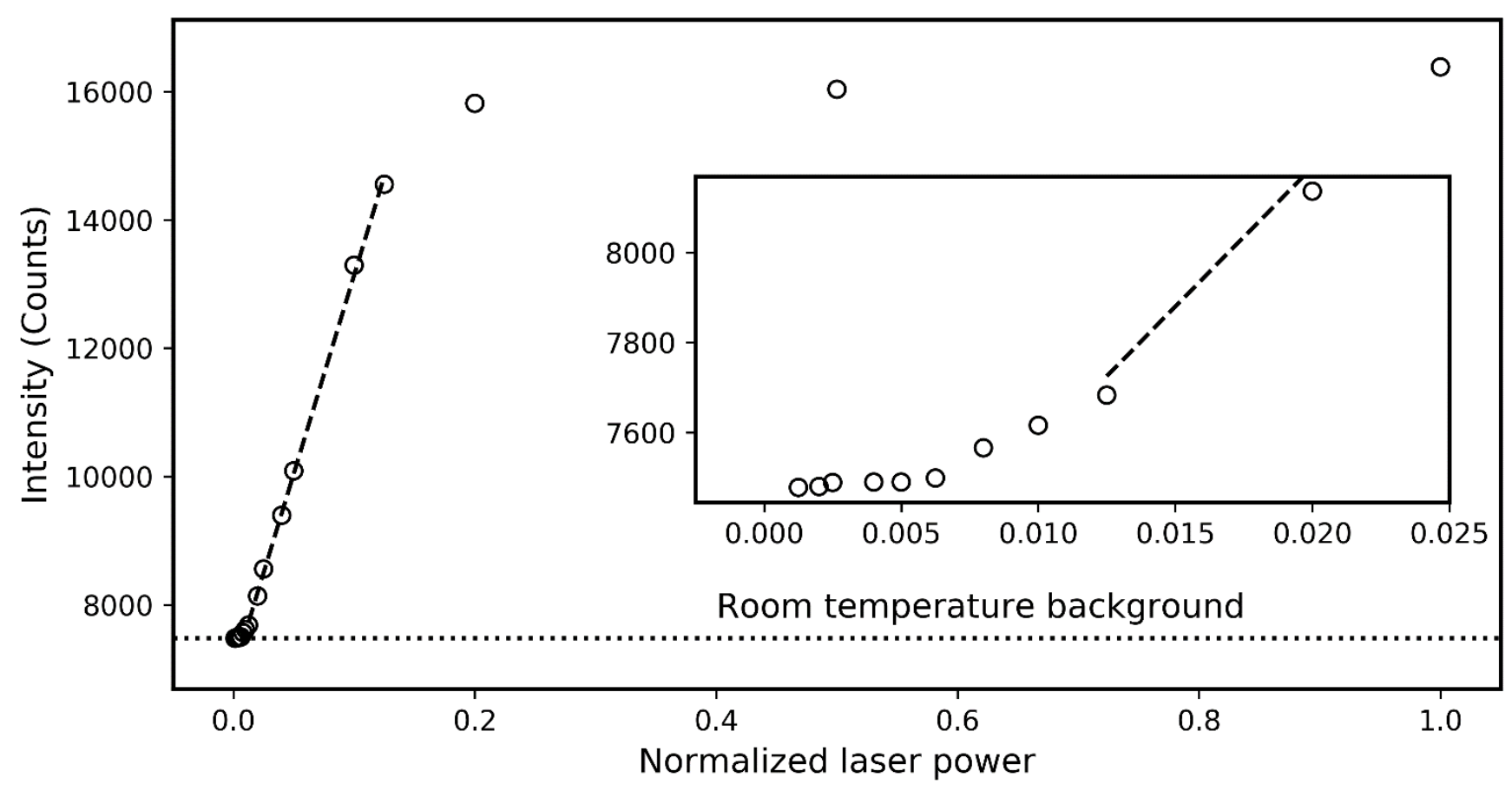

Figure 5. Plot of micro-bolometer intensity recorded using a $6 \mu \mathrm{m}$ beam at powers ranging from 1.9 to $1500 \mu \mathrm{W}$, pulse energy of $15 \mathrm{~nJ}$ and a spot size of $92 \mu \mathrm{m}$ (FWHM). The dashed line represents a linear fit to the data showing the range over which the device is linear. The dotted line shows the room temperature background detected by the bolometer array. The inset shows the first 10 data points on a larger scale, showing that below an intensity of ca 7,750 counts the response of the bolometer is not linear.

\section{Beam Profiling, Spatial Overlap and Beam Drift}

The bolometer array increases the speed and ease with which it is possible to identify aberration in either an infrared probe or pump beam, and saves time when determining the focal plane and focal spot-size (shown above). Figure 6 demonstrates how the device can easily identify aberration within a focal spot, in this instance induced by poor alignment of the infrared beam onto an off-axis parabolic mirror. As shown in figure 6, such aberration has a significant impact on the focal spot size and shape and only requires a small displacement of the beam as it approaches the focusing mirror. Figure 7 shows an image, at the focal plane, of the beam from the systems pulseshaper used for the pump beam in two-dimensional infrared experiments. This beam exhibits satellite artefacts along the $\mathrm{x}$-axis or vertical axis in the experimental setup. Without a means of viewing the infrared beam profile the detection of these effects would be both time consuming and difficult. Either effect could have a significant impact on any experimental signal and the quality of collected data; for example, it is feasible for the operator to overlap beams using one of the satellite beams rather than the main portion of the beam by mistake. 

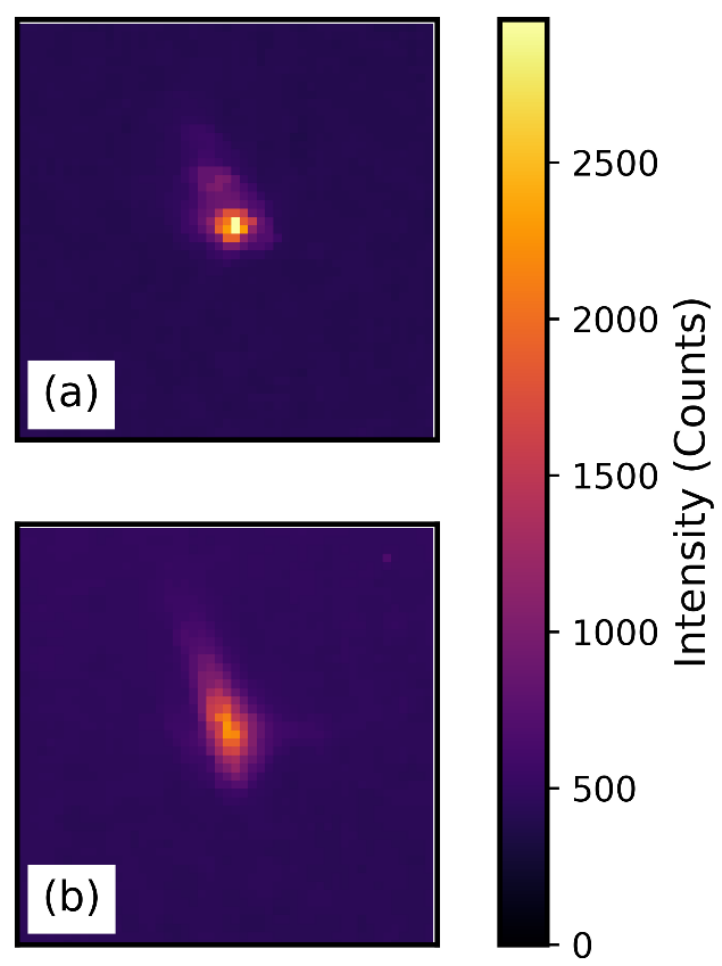

Figure 6. $50 \times 50$ pixel sections of the bolometer array containing the focus of a $4 \mu \mathrm{m}$ beam ( $20 \mathrm{~nJ}$ at 10 $\mathrm{kHz}$ ) with a small amount of aberration (a) and severe aberration (b). The image is the result of ca 1430 laser pulses.
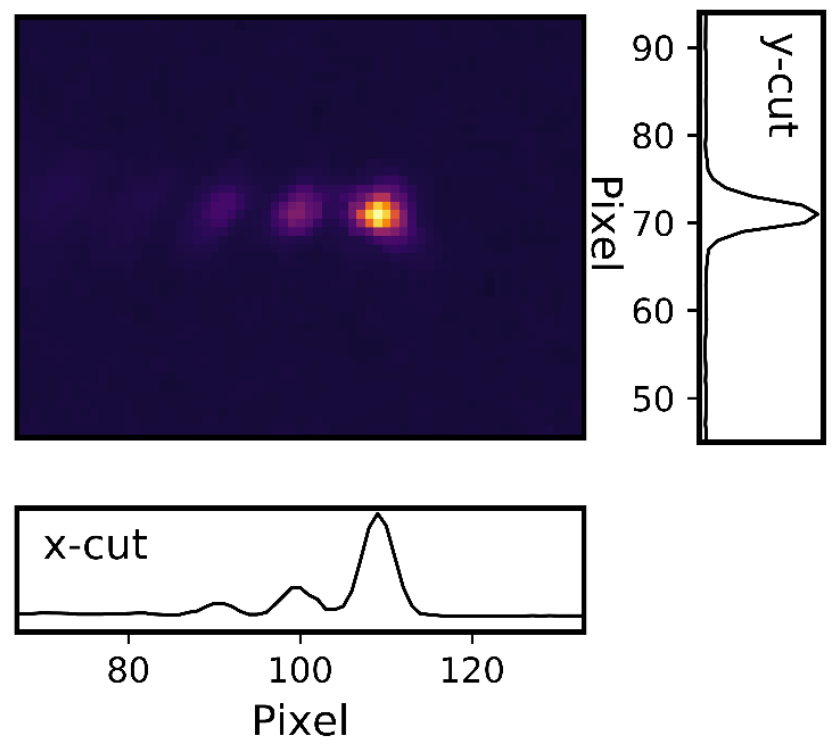

Figure 7. A $65 \times 49$ pixel section of the the micro-bolometer array illustrating artefacts in the form of satellite beams from the infrared pulse-shaper/optical delivery system, visible at the focal point of the beam. Laser wavelength is $6 \mu \mathrm{m}$, with a pulse energy of $60 \mathrm{~nJ}$ at $400 \mathrm{~Hz}$. The image is the result of approximately 60 laser pulses. 
Prior to purchasing the micro-bolometer, obtaining spatial overlap of multiple mid-infrared beams was a tedious task, requiring location of the focal point, overlap of the beams using a pinhole in the focal plane and spot-size measurement of each beam with a knife-edge. This would often require repetition numerous times after adjusting the collimation of the beams to achieve an optimal focal spot size. At best, this process took many hours. With the introduction of the micro-bolometer array to the laboratory the time-taken to achieve this task is vastly reduced making the task much easier, leaving more time for research. Figure 8 shows images from the bolometer array recorded as it travels along the optical axis. Each image shows three infrared beams which focus to the same point, at which all three beams are spatially overlapped.
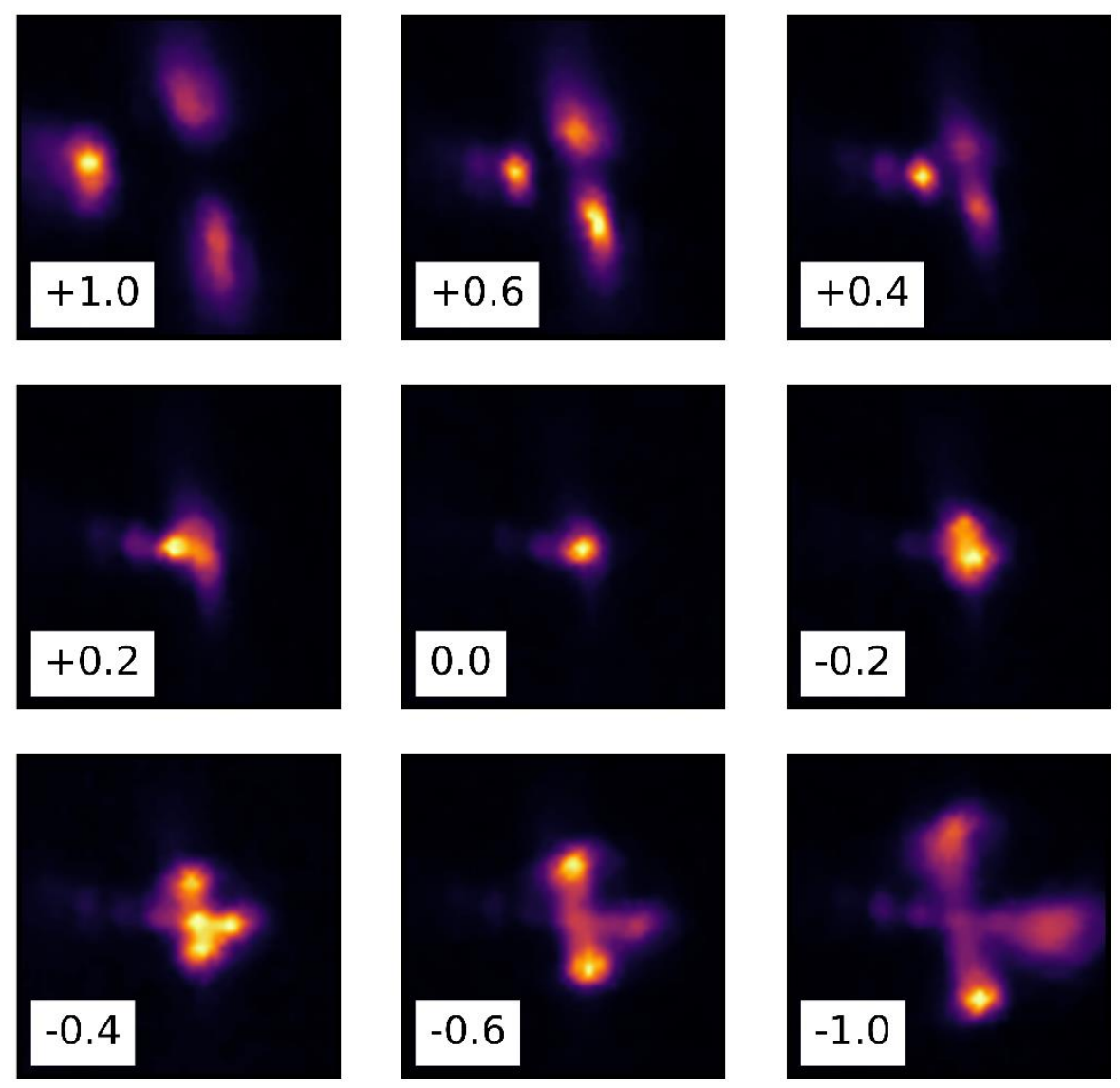

Figure 8. Images demonstrating the spatial overlapping of three infrared beams at the focal point of an off-axis parabolic mirror, $f=75 \mathrm{~mm}$, centre image. The beam produced by the pulse-shaper, has the satellite beams, shown in figure 7. Each image shows the processed camera output at a particular point along the experimental optical axis. The camera was translated along this axis between images, with the distance, in millimetres from the focal point indicated in each image. Each image, which displays a $50 \times 50$ pixel section of the bolometer array, is auto-scaled to use the full colour range for clearer display. 
The bolometer is also an extremely useful tool for monitoring long-term drifts in infrared beam position by capturing and analysing individual frames, a process which can easily be automated. As discussed above, it is not possible to trigger the device from an external source, and it has a relatively slow frame rate; it is therefore not possible to capture every laser shot. By recording every $4^{\text {th }}$ frame a frame capture rate of approximately $1.3 \mathrm{fps}$ is achieved. Fitting a two-dimensional Gaussian to each image enables tracking of the beam position with time. Figure 9 shows the drift over 15 minutes of one of the $6 \mu \mathrm{m}$ laser outputs from the LIFEtime system after opening its pulse-picker. There is a clear horizontal drift over time, whereas the vertical beam position is constant, with the exception of the point, marked by an arrow, when the door to the laboratory opened. Changes in the laboratory air pressure are known to affect the laser system, this effect is reduced by shielding around the laser; however, a small drift in beam position can be seen. It is worth noting that, the significance of a relatively small, $1.5 \mu \mathrm{m}$, drift is low given that the FWHM of the beam was $75 \mu \mathrm{m}$.

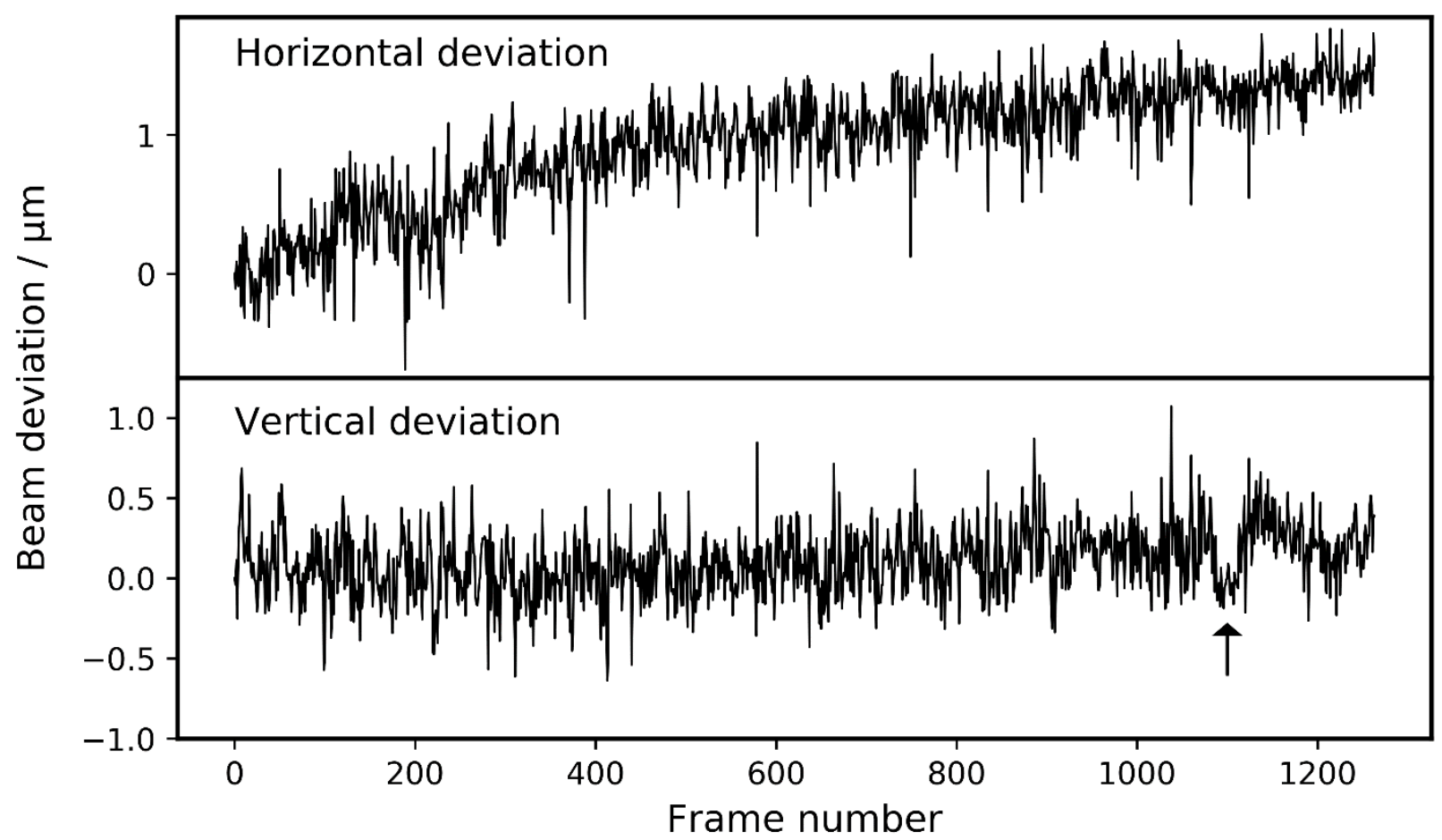

Figure 9. Plots of long-term beam deviation of the $6 \mu \mathrm{m}$ output of the laser system monitored at the focal plane. Each frame represents an average of approximately 7,500 laser shots, saving every $4^{\text {th }}$ frame gave a frame capture rate of ca $1.3 \mathrm{fps}$, total acquisition time is 15 minutes. Deviation in the horizontal plane (top) and vertical plane (bottom). The arrow indicates the point at which the laboratory door opened. 


\section{Spectrographic Detection}

As an array device there is potential to employ the micro-bolometer as a detector for infrared spectroscopy. The detectors usually employed for such applications are MCT focal plane arrays costing many tens of thousands of pounds. In order to test the potential of the bolometer array as a low-cost spectrographic detector, the data acquisition code required additional features, including a means of vertical binning of the pixels over a region of interest, frame averaging and operation in a 'chopped' mode using a shutter controlled by the software. In 'chopped' mode, each image is the result of two frames, one with infrared light on the detector and the other a dark frame, the two images are subtracted. For spectral acquisition, the calibration image was unused; its subtraction simply removes a baseline from recorded spectra.

The dimensions of a typical MCT focal plane array are $32 \times 1 \mathrm{~mm}$ for a 128-element array with $200 \mu \mathrm{m}$ wide pixels with a $50 \mu \mathrm{m}$ separation. The $206 \times 156$ element micro-bolometer sensor (unknown pixel separation) has an approximate width of $2.5 \mathrm{~mm}$, which is ca 13 times narrower than the MCT detector, but has ca 1.6 times more pixels. Clearly to see the same spectral bandwidth, careful grating choice is required. The results of the test using a ca $200 \mathrm{~cm}^{-1}$ bandwidth beam at $6 \mu \mathrm{m}$ and a 30 lines $/ \mathrm{mm}$ grating blazed at $8 \mu \mathrm{m}$ are shown in figure 10 . The figure shows the resulting image, binned data and calculated infrared spectrum using a polystyrene sample. A clear spectrum of polystyrene in the $1480 \mathrm{~cm}^{-1}$ region can be seen, showing $140 \mathrm{~cm}^{-1}$ bandwidth and ca $0.7 \mathrm{~cm}^{-1}$ per pixel. The fact the edges of the detector still show a significant amount of light indicates that the device was not capturing all the bandwidth, a $20 \mathrm{l} / \mathrm{mm}$ grating in the $125 \mathrm{~mm}$ spectrometer may have permitted the full bandwidth to be observed, nevertheless, the data shows clearly that the micro-bolometer can work well as a spectroscopic detector. 

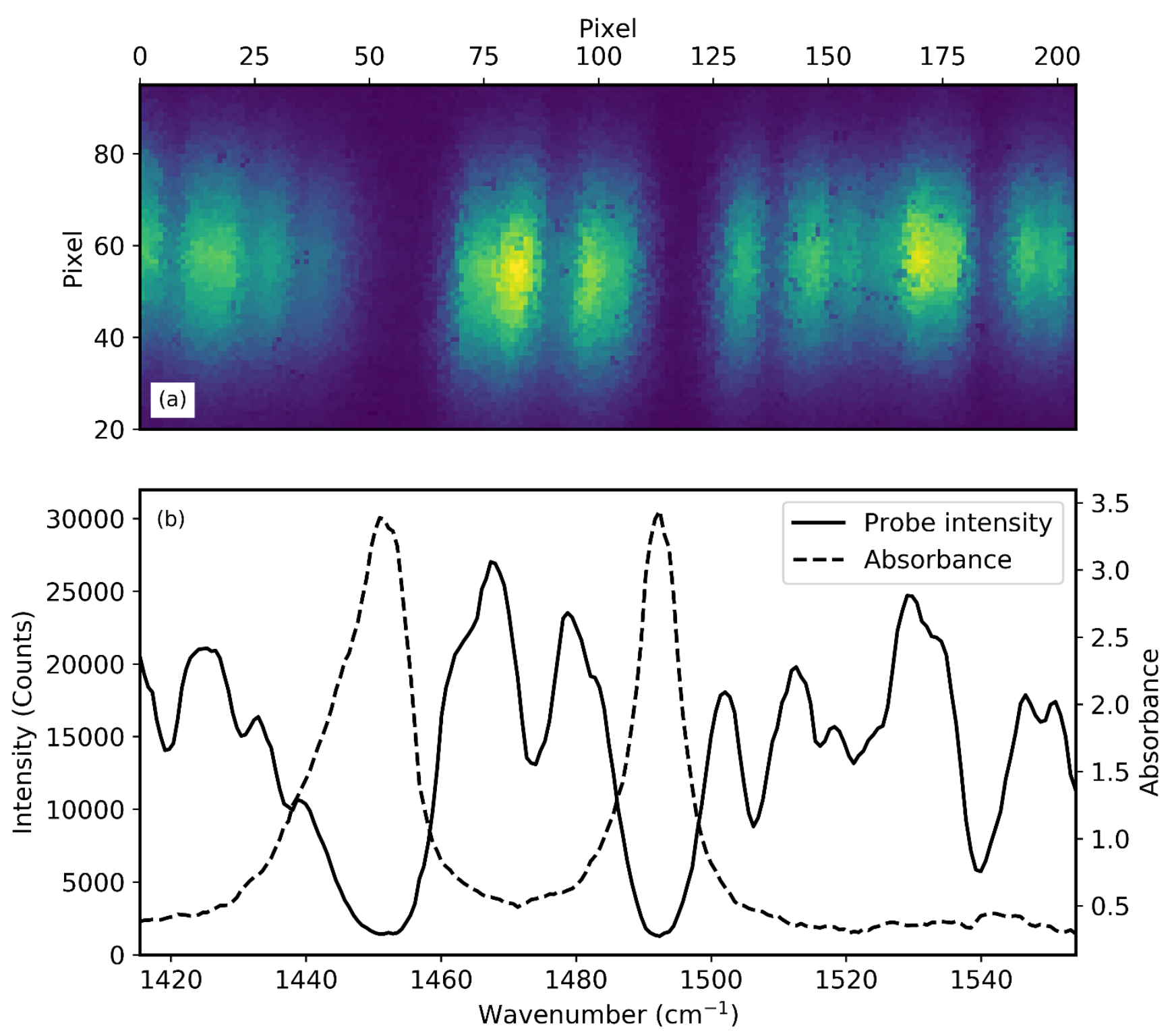

Figure 10. (a) Image showing the section of the micro-bolometer array illuminated by the infrared light with a $38 \mu \mathrm{m}$ polystyrene sample in the beam. The infrared light was dispersed using a $125 \mathrm{~mm}$ spectrograph (Oriel Instruments, model 77400 ) with a 30 lines $/ \mathrm{mm}$ grating blazed at $8 \mu \mathrm{m}$ and the detector was operated in 'chopped' mode. (b) Plot of the binned light intensity (binned over 75 pixels in the vertical direction) on the array (solid line), which clearly shows absorbance features from the polystyrene sample and water vapour lines. The dashed line shows the absorbance of the polystyrene sample.

\section{Conclusions}

The work presented shows how it is possible to use a low-cost compact off-the-shelf micro-bolometer array designed for mobile devices for a number of applications with infrared laser beams that can significantly ease various tasks with infrared lasers. These uses range from spot-size measurement, aberration and artefact 
detection, spatial overlap of beams, monitoring of long-term beam drift and use as a low-cost spectroscopic infrared array detector. The data presented shows that the device is sensitive in the range 1.3 to $10 \mu \mathrm{m}$, but we anticipate the upper limit of its usable range to be close to $20 \mu \mathrm{m}$. The supplier specifies the working range to be 7.5 to 14 $\mu \mathrm{m}$, but this is likely limited by the supplied chalcogenide lens. The use of such an infrared viewing aid can vastly reduce the amount of time required to set-up complex experiments involving infrared beams, being particularly useful for time-resolved spectroscopic techniques such as time-resolved infrared and two-dimensional infrared spectroscopies. The compactness of the device enables it to fit in even the smallest sample region.

One downside of the micro-bolometer array is that at present it is insensitive to light with a wavelength less than ca $1.3 \mu \mathrm{m}$ we are presently looking into whether or not this limitation can be overcome which would enable use of the device to overlap near- or mid-infrared beams with visible beams, particularly useful for pump-probe spectroscopic experiments. In addition, the room temperature background seen by the micro-bolometer limits its usable dynamic range.

It is possible the micro-bolometer will find a use in assisting in phase-correction of two-dimensional infrared spectra. The technique is particularly challenging, being sensitive to both spatial and temporal overlap of three beams when the impulsive time-domain technique is used. Using this approach requires sub-cycle accuracy in pulse timings and the experimentalist must know the absolute phase. Hamm and co-workers have reported a setup in which a pinhole is placed in the focal plane to induce scatter enabling the interference fringes of two pulsepairs to be observed.[14,15] Cundiff and colleagues have developed a similar technique for two-dimensional visible spectroscopy where a camera is placed in replica focal plane.[16] The micro-bolometer array may be able to work in a similar manner for two-dimensional infrared spectroscopy.

The fact that the micro-bolometer can be used as an infrared array detector for spectroscopic applications makes it particularly useful for quick test experiments, being very portable requiring just the detector and a computer. The low-cost of the device means that infrared array detectors should not be beyond the budget of teaching environments.

\section{Acknowledgements}


The authors gratefully acknowledge the support of the Science and Technology Facilities Council and the contributors to the post entitled "Yet another cheap thermal imager incoming.. Seek Thermal" on the EEVblog Electronics Community Forum,[17] without which the initial steps of acquiring raw data from the Seek microbolometer would have taken significantly longer.

\section{References}

1. Dickey, FM and Holswade SC, editors 2000 Laser Beam Shaping: Theory and Techniques (New York: Marcel Dekker, Inc).

2. Magnes J, Odera D, Hartke J, Fountain M, Florence L and Davis V 2006 Quantitative and qualitative study of Gaussian beam visualization techniques arXiv:physics/0605102.

3. Glover DM, Jenkins WJ and Doney SC 2011 Modelling methods for marine science, (New York: Cambridge University Press), p 398.

4. Kimpe T and Tuytschaever T, 2007 Increasing the number of gray shades in medical display systems How much is enough? Journal of Digital Imaging 20, 422.

5. Greetham GM, Donaldson PM, Nation C, Sazanovich IV, Clark IP, Shaw DJ, Parker AW and Towrie M 2016 A $100 \mathrm{kHz}$ time-resolved multiple-probe femtosecond to second infrared absorption spectrometer Applied Spectroscopy 70, 645.

6. Donaldson PM, Greetham GM, Shaw DJ, Parker AW and Towrie M 2018 A 100 kHz pulse shaping 2D-IR spectrometer based on dual Yb:KGW amplifiers Journal of Physical Chemistry A 122, 780.

7. Tulloh D, https://github.com/lod/seek-thermal-documentation/wiki/Frame\#basic-information.

8. Cignoli F, De luliis S and Zizak G 2004 A webcam as a light probe beam profiler Applied Spectroscopy $58,1372$.

9. Langer G, Hochreiner A, Burgholzer P and Berer T 2013 A webcam in Bayer-mode as a light beam profiler for the near infra-red Optics and Lasers in Engineering 51, 571.

10. Mitchell DP and Netravali AN 1988 Reconstruction filters in computer-graphics Proceedings of 15th Annual Conference on Computer Graphics and Interactive Techniques - SIGGRAPH '88 (New York: ACM Press), pp. 221-228.

11. Wrenninge M 2013 Production Volume Rendering: Design and Implementation (New York: CRC Press). 
12. Rogalski A 2002 Infrared detectors: an overview Infrared Physics and Technology 43, 187.

13. Niklaus F, Vieider C and Jakobsen H 2007 Proc. SPIE 6836, 68360D.

14. Backus EHG, Garrett-Roe S and Hamm P 2008 Phasing problem of heterodyne-detected twodimensional infrared spectroscopy Optics Letters 33, 2665.

15. Hamm P and Zanni M 2011 Concepts and Methods of 2D Infrared Spectroscopy (Cambridge: Cambridge University Press).

16. Bristow AD, Karaiskaj D, Dai X and Cundiff ST 2008 All-optical retrieval of the global phase for twodimensional Fourier-transform spectroscopy Optics Express 16, 18017.

17. EEVblog Electron. Community Forum (2014), https://www.eevblog.com/forum/thermal-imaging/yetanother-cheap-thermal-imager-incoming/ 